\title{
Magnetic pendulum apparatus for analog demonstration of first-order and second-order phase transitions and tricritical points
}

\author{
Authors: V. Hugo Schmidt \& B.R. Childers
}

Copyright (1984) American Institute of Physics. This article may be downloaded for personal use only. Any other use requires prior permission of the author and the American Institute of Physics. The following article appeared in American Journal of Physics, volume 52 and may be found at http://dx.doi.org/10.1119/1.13847.

V.H. Schmidt and B.R. Childers, "Magnetic pendulum apparatus for analog demonstration of firstorder and second-order phase transitions and tricritical points," American Journal of Physics. 52, 39-43 (1984).

http://dx.doi.org/10.1119/1.13847

Made available through Montana State University's ScholarWorks

scholarworks. montana.edu 


\title{
Magnetic pendulum apparatus for analog demonstration of first-order and second-order phase transitions and tricritical points
}

\author{
V. Hugo Schmidt and Bryan R. Childers \\ Physics Department, Montana State University, Bozeman, Montana 59717
}

(Received 26 July 1982; accepted for publication 1 April 1983)

\begin{abstract}
A simple apparatus is described in which the deflection of a pendulum with an electromagnet mounted at its tip is the analog of the magnitude of the order parameter in a phase transition. Its deflection is governed by the current through this electromagnet and currents through fixed electromagnets just below it. By suitable variation of these currents, the pendulum can be made to undergo gradual (second-order transition analog) or sudden (first-order transition analog) displacements from the vertical, as well as the intermediate situation corresponding to a tricritical point. Results for critical and tricritical exponents obtained with this apparatus are presented.
\end{abstract}

\section{INTRODUCTION}

The past decade has seen a considerable increase in interest in phase transitions and critical phenomena, owing largely to the success of renormalization group theory in describing phenomena associated with fluctuations of the order parameter of the transition. Pedagogical articles in this area are sparse enough that it is practical as well as appropriate to list all those found in a search of the American Journal of Physics. Demonstrations and student experiments include the ethane critical point, ${ }^{1}$ aniline-cyclohexane mutual solubility critical point, ${ }^{2}$ nitrogen freezing, ${ }^{3}$ a projection demonstration with interference colors of the solid-solid transitions of $\mathrm{NH}_{4} \mathrm{NO}_{3},{ }^{4}$ metallic glass-crystalline transition, ${ }^{5}$ several magnetic transitions, ${ }^{6-9}$ and the two ferroelectric transitions in Rochelle salt. ${ }^{10}$

Expository theoretical articles include a one-dimensional model for $\mathrm{KH}_{2} \mathrm{PO}_{4}$ exhibiting a first-order phase transition, ${ }^{11}$ a discussion of first- and second-order phase transitions aided by geometrical arguments which employs a second-order ferroelectric transition as an example, ${ }^{12}$ a magnetic model having a first-order phase transition which exhibits both stable and metastable states, ${ }^{13}$ a calculation of critical exponents from percolation clusters, ${ }^{14}$ an exactly soluble model exhibiting a Landau phase transition with a discussion of fluctuation effects, ${ }^{15}$ and an article on how to teach the renormalization group. ${ }^{16}$ Finally, in the spirit of the present article, a mechanical model for the spin-fiop transition in an antiferromagnet has been described. ${ }^{17}$

If one wishes to demonstrate, or have students measure, 
quantitative phenomena associated with phase transitions, several difficulties arise. First, considerable apparatus is required to slowly vary the temperature through the transition, and this variation is too slow to be practical for a demonstration experiment. Second, additional apparatus is needed to measure the magnitude of the order parameter (magnetic or electric polarization, density change, etc.) associated with the phase transition. This apparatus was constructed to provide an easily visible and rapidly adjustable analog of the transition order parameter.

\section{APPARATUS DESCRIPTION}

The apparatus consists basically of a pendulum with an electromagnet at its tip which is in close proximity to three equally spaced fixed electromagnets, as shown in Fig. 1.

The pendulum and its support are a Cenco two-pan balance with pans removed, and with additions described below. We placed the balance on a rigid steel box high enough so that the pendulum bob would just clear the fixed electromagnets. Low pivot friction is essential for sensitive operation of this apparatus; the pivot of this Cenco balance is a steel knife edge resting on two glass $V$ blocks. The short rod holding these $\mathrm{V}$ blocks was reversed in its clamp at the top of the balance support so that the $V$ blocks stick out beyond the back of the support base, allowing the pendulum to clear the base.

Going from top to bottom, the pendulum consists of the Cenco pivot arm with pointer rod unscrewed and replaced by a machine screw onto which an aluminum collar was screwed, as shown in Fig. 2. A stainless steel rod $68.5 \mathrm{~cm}$ long was inserted into this collar and held with a set screw. Another aluminum collar at the bottom of this rod connects this rod to the electromagnet core. This steel core is $0.375 \mathrm{in} .(10 \mathrm{~mm})$ in diameter and $6.5 \mathrm{in} .(16.5 \mathrm{~cm})$ long, and is the core of an old Welch mutual inductance apparatus whose outer coil has a base 1.75 in. $(44 \mathrm{~mm})$ square. The

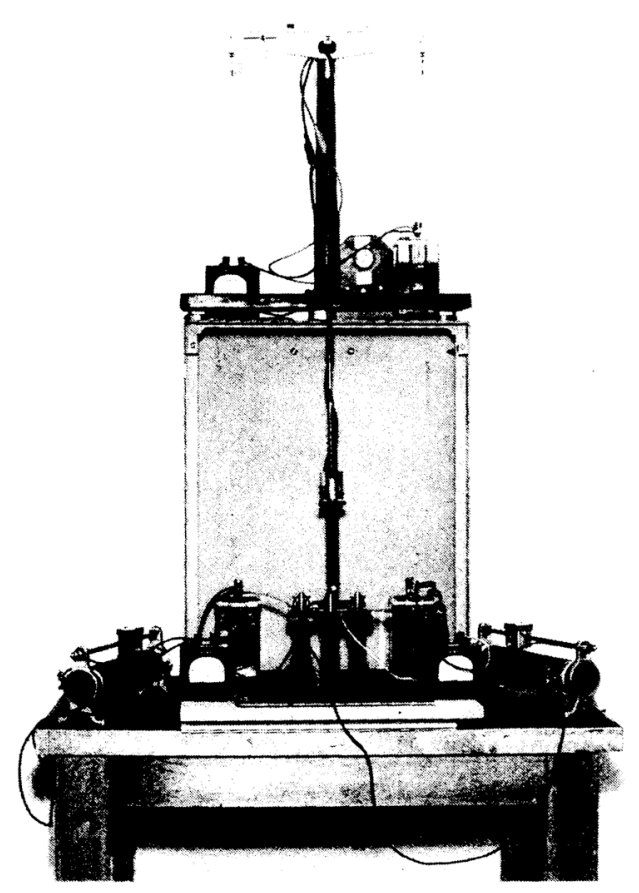

Fig. 1. Photograph of the version of the apparatus employing batteries rather than power supplies.
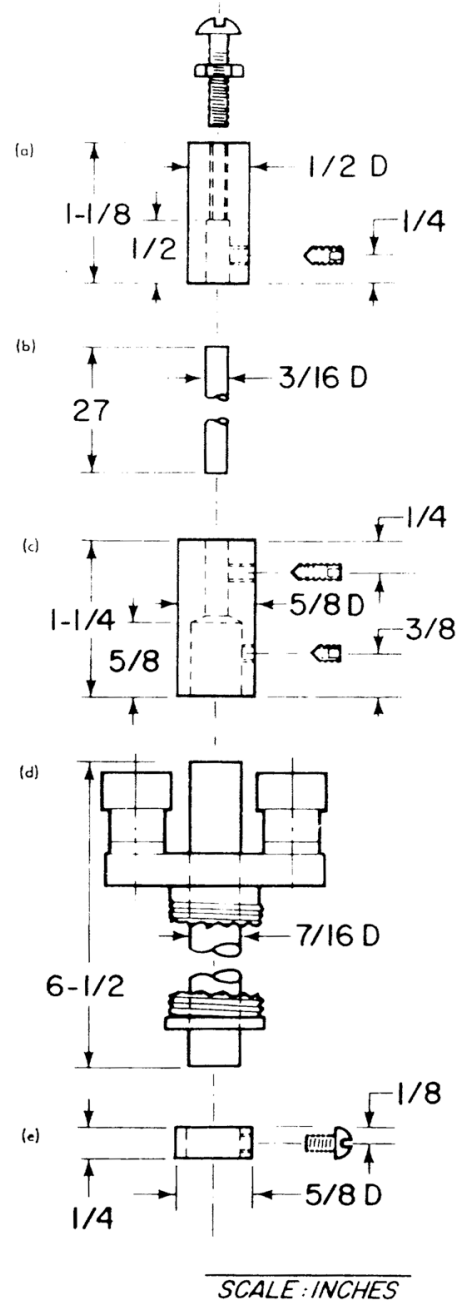

Fig. 2. Exploded view of pendulum construction. (a) Aluminum collar drilled and threaded for an 8-32 screw and lock nut which attach the pendulum to the balance crossbar, and drilled also for the pendulum rod which is held in place by a 6-32 set screw. (b) Stainless steel pendulum rod. (c) Aluminum collar drilled for the pendulum rod and the electromagnet core, each of which is held in place with a 6-32 set screw. (d) Permeable steel electromagnet core and its surrounding coil to which connections are made by means of the two banana jacks shown. (e) Aluminum collar on which the electromagnet coil rests; this collar is held in place 1/16 in. above the bottom of the electromagnet core by a nonmagnetic 6-32 screw. Dimensions are given in inches.

inner coil of this apparatus is slipped over the core with its terminals on top. It is held in place by a thin aluminum collar which is held onto the lower end of the core by a nonmagnetic screw. This inner coil is wound over a brass cylinder which fits loosely over the core. It has 180 turns of approximately 1-mm-diam insulated wire wound in two layers. The two leads to this coil are fine insulated wires which are run up along the pendulum rod and through holes in the Cenco pivot arm near the pivot.

We contrived a simple device (not pictured) for measuring the pendulum deflection. A meter stick was fastened to the metal box support, parallel to the Cenco base and slightly above the electrical connections to the pendulum electromagnet. A small rectangular mirror was taped to the reverse side of the meter stick such that the image of the pendulum rod could be observed. The electromagnet wires 
were spaced away from the rod. By visually aligning the pendulum rod with its reflection we obtained readings accurate to within $0.02 \mathrm{~cm}$.

The fixed electromagnets below the pendulum are similar old-style Welch mutual inductance demonstration devices. The outer coil frame is mounted on a base 1.75 in. $(4.44 \mathrm{~cm})$ square. These three square bases are butted up against each other, in line with the pendulum swing, with the center electromagnet just below the pendulum equilibrium position. They are held together with two rubber bands that encircle all three electromagnets, to increase stability. Inside each of these coils is a mild steel core, identical to the one on the pendulum. The cores are shimmed with electrical tape to prevent movement of the cores from influencing the experiment.

Although we used various blocks to adjust the air gap between the electromagnets, a better way to vary this parameter is to mount the three stationary magnets on a laboratory jack. The air gap can then be adjusted without disturbing the alignment.

The electrical arrangement consists of four separate circuits, each being a simple series circuit consisting of an adjustable, regulated, low-voltage, high-current power supply, an ammeter, and one or two electromagnets. Two of the circuits drive the pendulum electromagnet and the outer coil of the center fixed electromagnet, respectively. The third circuit drives the inner coils of the outer two fixed magnets which are connected in series with the same polarity (say N), but opposite to the polarity $(\mathrm{S})$ of the center fixed electromagnet and pendulum electromagnet. (These coils are similar to the pendulum magnet coil.) The fourth circuit drives the outer coils of the two outer fixed electromagnets. (Each outer coil has about 1200 turns wound in six layers.) They are used to create a bias to one side to simulate the effect of an ordering field (electric field for ferroelectric phase transitions). Although we found better success with the fourth circuit a similar effect may be produced by slightly tilting the apparatus. Finally, the balance should be adjusted so that the pendulum electromagnet is positioned directly over the center electromagnet and there is no bias favoring either direction of pendulum deflection.

\section{DEMONSTRATION OF TRANSITION ORDER AND CRITICAL EXPONENTS}

The operation of this device can be explained most easily in terms of a potential energy function $V(\theta)$ for the pendulum, which is the analog of the Helmholtz free energy $F(P)$, where $P$ is the transition order parameter, for instance electric polarization for a ferroelectric phase transition. Such ferroelectric transitions are discussed by Lines and Glass, ${ }^{18}$ while a more introductory treatment of phase transition in general is given by Stanley. ${ }^{19}$ Mean-field theory predictions for tricritical points and other critical phenomena in magnetic materials were reviewed by Kincaid and Cohen. ${ }^{20}$

To demonstrate a second-order phase transition, it is not necessary to energize the outer two fixed electromagnets; however, it is advisable to remove the iron cores from these coils to prevent induced magnetization from altering the data. With no power applied to any magnets, $V(\theta)$ is just the gravitational potential energy for the pendulum, which is minimum for $\theta=0$. As current is increased in both the pendulum magnet and center fixed magnet, their mutual repulsion reduces the curvature of $V(\theta)$ evaluated at $\theta=0$. When the curvature vanishes, the pendulum will start to move to one side or the other; this situation corresponds to the transition temperature at which the order parameter $P$ starts to change smoothly away from zero. A critical exponent can be determined from the relation between $I-I_{c}$ and $\theta$ for conditions under which there is no jump of $\theta$; here $I$ is the current in the pendulum magnet and $I_{c}$ is the critical current at which displacement begins. This is the analog of $P \propto\left(T_{c}-T\right)^{\beta}$, where $T_{c}$ is the transition temperature and $\beta$ is the critical exponent which, in mean field theory, has the value $\frac{1}{2}$ for a critical point and $\frac{1}{4}$ for a tricritical point. For small deflections a linear measure of the deflection will yield, on a log-log graph of displacement versus $\left(I-I_{c}\right)$, the value of $\beta$, as shown in Fig. 3 .

To demonstrate a first-order phase transition, for which the order parameter $P$ jumps suddenly from zero to a finite value as temperature is lowered, it is necessary to energize also the outer fixed coils. If their current is large enough compared to the current in the center fixed coil, the pendulum will jump suddenly from $\theta=0$ to the vicinity of one of the fixed outer coils as the pendulum magnet current is increased smoothly. As this current is increased slowly from zero under these conditions (the analog of decreasing the temperature), $V(\theta)$, which starts with a single central minimum, develops two equally spaced side minima at nonzero $\theta$. As current is increased, these minima become lower than the central minimum, which becomes a position of metastable equilibrium until the local curvature vanishes, at which time the jump will occur. (This differs from the behavior for a first-order phase transition in that the system need not stay in the metastable minimum until that minimum vanishes, but rather can jump as soon as the side minima become lower than the central minimum.)

The tricritical point occurs when a second parameter, such as hydrostatic pressure, is adjusted simultaneously

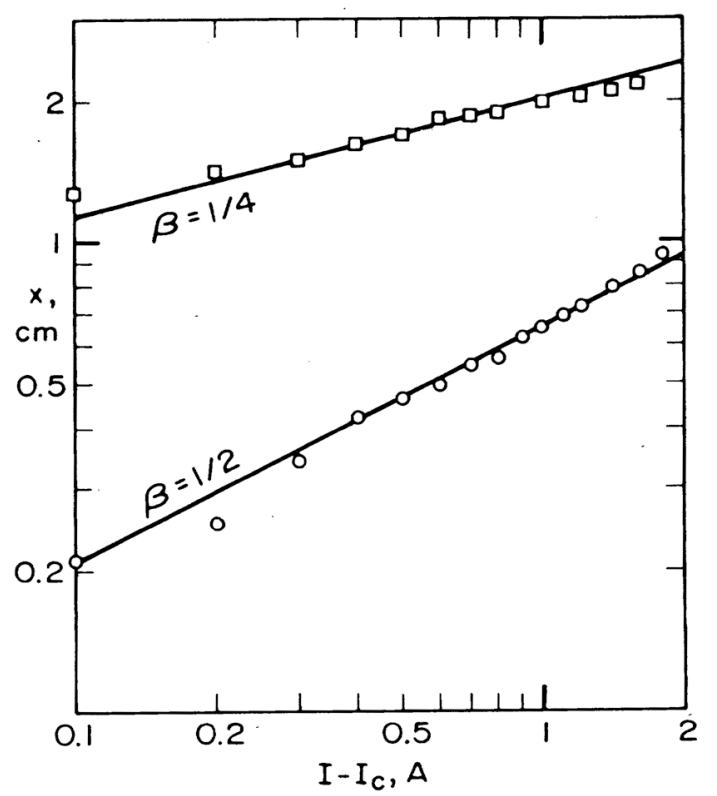

Fig. 3. Data for critical and tricritical exponent $\beta$. Here $x$ is the pendulum deflection read about $2 / 3$ of the way from the pivot to the magnet tip, and $I-I_{c}$ is the pendulum magnet current less its critical value. For the critical exponent data (circles) the center magnet current was $0.72 \mathrm{~A}$ and the air gap was $9 \mathrm{~mm}$. For the tricritical exponent data (squares) the current was $0.06 \mathrm{~A}$ in the center magnet and $5.0 \mathrm{~A}$ in the outer magnets, and the air gap was $12 \mathrm{~mm}$. 


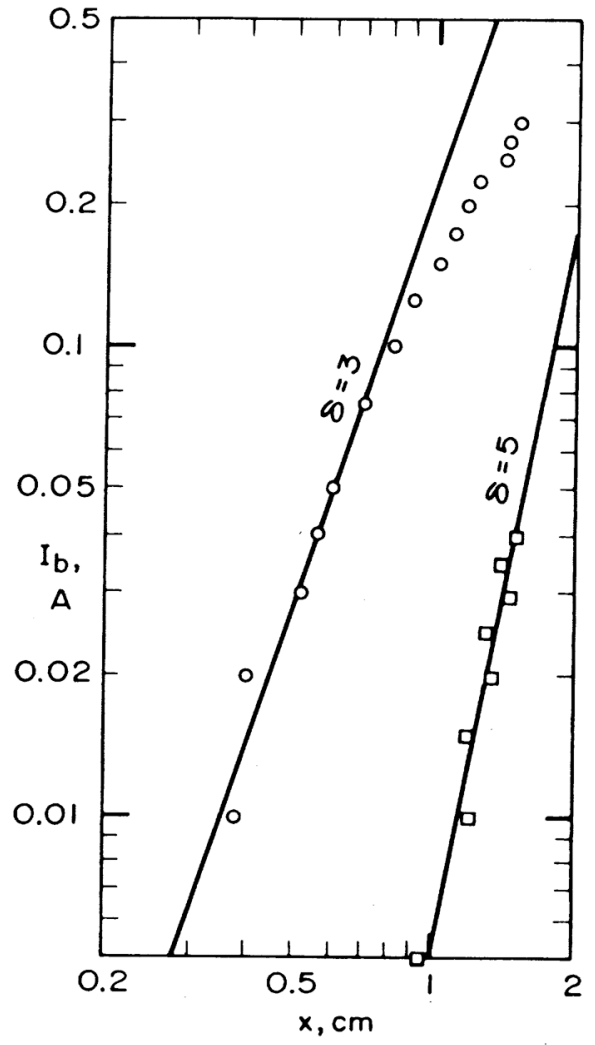

Fig. 4. Data for critical and tricritical exponent $\delta$. Here $x$ is pendulum deflection and $I_{b}$ is bias current in the outer coils of the outer magnet. For the critical exponent data (circles) the current was $3.0 \mathrm{~A}$ in the pendulum magnet and $0.53 \mathrm{~A}$ in the center magnet, with an 8- $\mathrm{mm}$ air gap. For the tricritical exponent data (squares) the current was $5.0 \mathrm{~A}$ in the pendulum magnet, $0.04 \mathrm{~A}$ in the center magnet, and 4.8 $\mathrm{A}$ in the inner coils of the outer magnets, with a 14-mm air gap.

with temperature in a system in which such adjustment can make the jump of the order parameter at the transition temperature vanish. For example, at a certain pressure the first-order ferroelectric phase transition in $\mathrm{KH}_{2} \mathrm{PO}_{4}$ becomes second order. ${ }^{21}$ The analog of this behavior is demonstrated in this apparatus by reducing the current in the outer coils until the jump in $\theta$ just vanishes. For this situation, the fourth as well as the second derivative of $V$ with respect to $\theta$ vanishes when the pendulum starts to move, so $V(\theta)$ is very flat and it is especially important to have the apparatus properly leveled.

As was noted earlier, the fourth circuit is used to simulate the effect of an ordering field. At the critical pendulum magnet current (temperature) the relation between bias magnet current (field) and pendulum displacement (order parameter) will obey a critical exponent relation, which for ferroelectrics in the Landau mean field theory ${ }^{18,19}$ is $E \propto P^{\delta}$, with the critical exponent $\delta$ being 3 for a secondorder transition (critical point) and 5 for a tricritical point as was experimentally observed for $\mathrm{KH}_{2} \mathrm{PO}_{4} \cdot{ }^{22}$ Our data for the critical and tricritical exponent $\delta$ appear in Fig. 4.

The bias created by the fourth circuit can also be used to find, graphically, the Curie-Weiss constant. For the Helmholtz free energy function $F$ we have

$$
F=U(S, P)-T S
$$

or, using the Landau expansion .

$$
F=A P^{2} / 2+B P^{4} / 4+C P^{6} / 6+\cdots,
$$

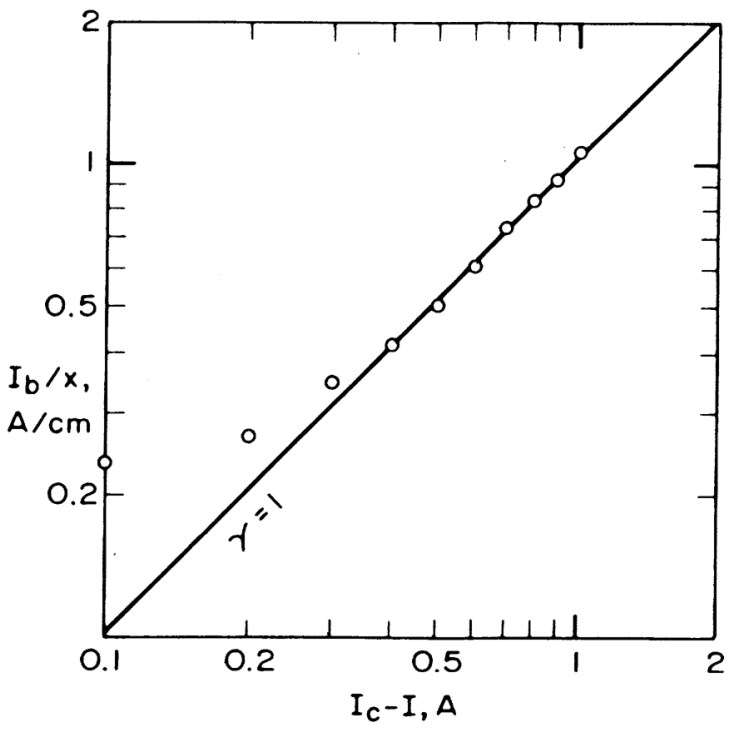

Fig. 5. Data for critical exponent $\gamma$. Here $I_{b} / x$ is bias current in the outer coils of the outer magnets divided by pendulum deflection, and $I_{c}-I$ is critical pendulum current less actual current. The current was $0.59 \mathrm{~A}$ in the center magnet and $2.0 \mathrm{~A}$ in the inner coils of the outer magnets, while the air gap was $9 \mathrm{~mm}$.

where $A=A_{0}\left(T-T_{0}\right), T_{0}$ being the Curie-Weiss temperature. But,

$$
E=\partial F / \partial P=A P+B P^{3}+C P^{5}+\cdots .
$$

Neglecting terms of order 3 and above because $E$ is small results in

$$
E=A_{0}\left(T-T_{0}\right) P .
$$

But we also have

$$
\chi=P / E=1 / A_{0}\left(T-T_{0}\right)
$$

where $A_{0}{ }^{-1}$ is the Curie-Weiss constant. In terms of the critical exponent $\gamma$, we have $\chi^{-1} \propto\left(T-T_{0}\right)^{\gamma}$, where $\gamma=1$ in this mean-field theory. A graph on log-log paper of bias current/deflection versus $I_{c}-I$ for pendulum transition current $I_{c}$ and pendulum current $I$ yielded a $\gamma$ exponent of 1. We found that the best method was to adjust the bias for about a 2-mm deflection of the pendulum for varying pendulum currents (see Fig. 5).

\section{DISCUSSION}

Several words of advice are offered on the basis of our experience with this apparatus. (1) The critical exponents are relatively easy to obtain, but the adjustments required to get good data for the tricritical exponents are very delicate and such data probably cannot be obtained in a single laboratory session by students. (2) If the gap between the pendulum magnet and the center fixed magnet is too small, there will be distortions in the potential energy function for small values of $\theta$. The gap should be made as large as possible consistent with being able to get sufficiently large deflections for currents small enough so that the magnet coils are not overheated. (3) If the pendulum magnet current is too large relative to the center magnet current, increasing the pendulum magnet current will not cause this magnet to be repelled from the center magnet, because an opposite pole will be induced in the center magnet. (4) Particularly for the tricritical measurements, we experienced consider- 
able difficulty with the effects of hysteresis in the magnet cores which would change the delicate equilibrium needed. These cores seem to be made of ordinary mild steel; a special steel with very thin hysteresis loops would be preferable.

Effects of some of the above difficulties can be seen in Figs. 3-5. Potential energy distortions at small $\theta$ as discussed in (2) above are responsible for the deviations at small currents in Figs. 3 and 5. To minimize hysteresis effects for the tricritical $\delta$ exponent data in Fig. 4, the bias current was reversed each time its magnitude was increased to obtain a new data point. The staggered arrangement of the data points results from a slight tendency of the pendulum to prefer to deflect in one direction which remained after the apparatus was equalized as accurately as possible. The deviations for large currents in Figs. 3 and 4 are to be expected and occur also in actual critical phenomena experiments, because the exponents only describe behavior very close to the critical or tricritical point.

This apparatus can provide an interesting demonstration in a statistical mechanics course or can be employed in an advanced undergraduate or graduate laboratory experiment which can be done much more quickly and easily than the corresponding experiment on a system with an actual phase transition. Analysis of results to determine critical and tricritical exponents can be particularly instructive. The required apparatus can be assembled quickly from student laboratory equipment available in most physics departments, and only a few simple parts need to be machined.

\section{ACKNOWLEDGMENT}

Rufus Cone of this Department kindly provided the photograph of this apparatus.

${ }^{1}$ B. H. Sage and H. H. Reamer, Am. J. Phys. 9, 310 (1941).

${ }^{2}$ L. White, Jr., Am. J. Phys. 34, 68 (1966).

${ }^{3}$ R. L. Wild and D. C. McCollum, Am. J. Phys. 35, 540 (1967).

${ }^{4}$ C. L. Miles, Am. J. Phys. 43, 650 (1975).

${ }^{5}$ P. M. Anderson, III, and A. E. Lord, Jr., Am. J. Phys. 46, 80 (1978)

${ }^{6}$ E. V. Smith, Am. J. Phys. 31, 731 (1963).

${ }^{7}$ W. T. Oosterhuis, Am. J. Phys. 31, 132 (1963).

${ }^{8}$ D. Prescetti, Am. J. Phys. 37, 334 (1969).

${ }^{9}$ B. R. Sood, R. Hamal, and S. Sikri, Am. J. Phys. 48, 481 (1980).

${ }^{10}$ V. H. Schmidt, Am. J. Phys. 37, 351 (1969).

"IJ. F. Nagle, Am. J. Phys. 36, 1114 (1968).

${ }^{12}$ G. Fischer, Am. J. Phys. 25, 100 (1957).

${ }^{13}$ J. I. Kaplan and H. Stenschke, Am. J. Phys. 38, 1323 (1970).

${ }^{14}$ D. Stauffer, Am. J. Phys. 45, 1001 (1977).

${ }^{15}$ R. Alben, Am. J. Phys. 40, 3 (1972).

${ }^{16}$ H. J. Maris and L. P. Kadanoff, Am. J. Phys. 46, 652 (1978).

${ }^{17}$ T. Bernstein, Am. J. Phys. 39, 832 (1971).

${ }^{18}$ M. E. Lines and A. M. Glass, Principles and Applications of Ferroelectrics and Related Materials (Clarendon, Oxford, 1977).

${ }^{19} \mathrm{H}$. E. Stanley, Introduction to Phase Transitions and Critical Phenomena (Oxford U. P., New York, 1971).

${ }^{20}$ J. M. Kincaid and E. D. G. Cohen, Phys. Rep. 22, 57 (1975).

${ }^{21}$ V. H. Schmidt, A. B. Western, and A. G. Baker, Phys. Rev. Lett. 37, 839 (1976).

${ }^{22}$ A. B. Western, A. G. Baker, C. R. Bacon, and V. H. Schmidt, Phys. Rev. B 17, 4461 (1978). 